\title{
Mass at rest after quantum information
}

\author{
Vasil Penchev, vasildinev@gmail.com \\ Bulgarian Academy of Sciences: Institute of Philosophy and Sociology: \\ Dept. of logical Systems and Models
}

\begin{abstract}
The way, in which quantum information can unify quantum mechanics (and therefore the Standard model) and general relativity, is investigated. Quantum information is defined as the generalization of the concept of information as to the choice among infinite sets of alternatives. Relevantly, the axiom of choice is necessary in general. The unit of quantum information, a qubit is interpreted as a relevant elementary choice among an infinite set of alternatives generalizing that of a bit. The invariance to the axiom of choice shared by quantum mechanics is introduced: It constitutes quantum information as the relation of any state unorderable in principle (e.g. any coherent quantum state before measurement) and the same state already well-ordered (e.g. the well-ordered statistical ensemble of the measurement of the quantum system at issue). This allows of equating the classical and quantum time correspondingly as the well-ordering of any physical quantity or quantities and their coherent superposition. That equating is interpretable as the isomorphism of Minkowski space and Hilbert space. Quantum information is the structure interpretable in both ways and thus underlying their unification. Its deformation is representable correspondingly as gravitation in the deformed pseudoRiemannian space of general relativity and the entanglement of two or more quantum systems. The Standard model studies a single quantum system and thus privileges a single reference frame turning out to be inertial for the generalized symmetry U(1)XSU(2)XSU(3) "gauging" the Standard model. As the Standard model refers to a single quantum system, it is necessarily linear and thus the corresponding privileged reference frame is necessary inertial. The Higgs mechanism $U(1) \rightarrow U(1) X S U(2)$ confirmed enough already experimentally describes exactly the choice of the initial position of a privileged reference frame as the corresponding breaking of the symmetry. The Standard model defines 'mass at rest' linearly and absolutely, but general relativity nonlinearly and relatively. The "Big Bang" hypothesis is additional interpreting that position as that of the "Big Bang". It serves also in order to reconcile the linear Standard model in the singularity of the "Big Bang" with the observed nonlinearity of the further expansion of the universe described very well by general relativity. Quantum information links the Standard model and general relativity in another way by mediation of entanglement. The linearity and absoluteness of the former and the nonlinearity and relativeness of the latter can be considered as the relation of a whole and the same whole divided into parts entangled in general.
\end{abstract}

Keywords: general relativity, the Standard model, quantum information, mass at rest, qubit, the Big Bang 
There are two big and exceptionally corroborated theories about the fundamental physical reality. Unfortunately, they seem to be inconsistent with each other. General relativity explained very easily or even granted mass at rest. Quantum mechanics managed to confirm experimentally an analogical mechanism about it only almost a century later.

However if they are inconsistent, should the corresponding concept of mass at rest in each of them be one and the same? Or the opposed: might 'mass at rest' reconcile both great theories?

These two questions are investigated only in a possible reference frame: that of theory of quantum information (designated in the title and bellow abbreviated as "quantum information"); even less, only within a most common philosophical consideration referring to its foundation, principles and conditions.

The main conclusions of the article are: The concepts of mass at rest both in the Standard model and general relativity coincide being defined and a relevant relation in both cases. However, the Standard model defines that relation as linear and absolute while general relativity as non-linear and relative. Thus the former refers to some singularity or quantum leap while the latter to some smooth development of some whole consisting of parts. The additional hypothesis of the "Big Bang" interprets these differences in definition rather reasonably situating the origin of the Standard model in the singularity of the beginning of the universe, but referring the non-linear and relative approach of general relativity to the further development of the universe. Quantum mechanics also allows these two absolutely opposite viewpoints to be reconciled and even equated in the framework of wave-particle duality identifying the "Big Bang" and the further expansion of the universe as one and the same. Quantum information is the conceptual apparatus for that duality to be investigated relevantly and quantitatively within the theory of information for the concept of quantum information both generalizes that of information and unifies mathematically the discrete (quantum) and continuous (smooth). Furthermore, there are other possibilities for the consistent interpretation of 'mass at rest' in both great theories, being inconsistent in turn to the "Big Bang" hypothesis, but consistent to the approach of quantum information.

The plan of the paper is the following:

1. Quantum information: origin and concepts

2. The Standard model in terms of quantum information: mass at rest

3. General relativity in terms of quantum information: mass at rest

4. Mass at rest in quantum information and those in the Standard model and general relativity as its interpretations

5. Conclusions about the mathematical fundament of the physical world

\section{Quantum information: origin and concepts}

The first paper, which is usually cited in publications in quantum information, is Einstein. Podolsky, and Rosen's "Can Quantum-Mechanical Description of Physical Reality Be Considered Complete?" (1935). It was a culmination of Einstein's resistance against quantum mechanics just for relativity both special and general seemed inconsistent with it. The paper managed to demonstrate for first time that a new kind of correlations, the quantum correlations are implied by the mathematical 
formalism of quantum mechanics therefore restricting in addition the specific degrees of quantum freedom: the Heisenberg principle of uncertainty. The conclusion, which the three authors made, was that quantum mechanics is incomplete as it implies the existence of restrictions, which does not describe.

In fact, the theory of quantum information in the framework of quantum mechanics was generated just by the direct collision with relativity being due to their mutual inconsistency. In thus, the concept of quantum information can be very useful in order to elucidate interlinks between the notions and quantities about 'mass at rest' in both theories. The nature of that connection turns out to be a kind of generalization of the concept of information, namely quantum information.

The most important stages in the development and establishment of quantum information outlined the experimentally observable difference between "classical" and quantum correlations as well as the phenomena, in which they take place: those of entanglement.

The fundamental notion of quantum information is that of quantum bit, or 'qubit'. It is defined as follows:

'Qubit' is: $\alpha|0\rangle+\beta|1\rangle$ where $\alpha, \beta$ are complex numbers such that $|\alpha|^{2}+|\beta|^{2}=1$, and $|0\rangle,|1\rangle$ are any two orthonormal vectors (e.g. the orthonormal bases of any two subspaces) in any vector space (e.g. Hilbert space, Euclidean space, etc.)Thus Hilbert space underlying quantum mechanics is representable as the quantity of quantum information and any wave function, i.e. any state of any quantum system being a point in it can be seen as a value of that quantity. Consequently all physical processes turn out to be quantum-informational, and nature or the universe is a quantum computer processing quantum information

The qubit is also isomorphic to a ball in Euclidean space, in which two points are chosen: A qubit is equivalently representable as a unit ball in Euclidean space and two points, the one chosen within the ball, and the other being the orthogonal projection on its surface, i.e. as a mapping of a unit ball onto its surface (or any other unit sphere). Indeed: $\alpha, \beta$ are two complex numbers such that $|\alpha|^{2}+|\beta|^{2}=1 ;|\mathbf{0}\rangle,|\mathbf{1}\rangle$ are two orthonormal vectors or a basis such as two orthogonal great circles of the unit ball. Then: $\overrightarrow{\alpha|0\rangle}$ defines a point of the unit ball and the pair of $\overrightarrow{\alpha|0\rangle}$ and $\overrightarrow{\beta|1\rangle}$ defines a point of the unit sphere.

Consequently, Minkowski space, which is the space-time of special relativity, being an expanding ball in Euclidean space, can be understood as the same quantity of quantum information in another hypostasis of it. Therefore the concept of quantum information connects Hilbert space of quantum mechanics with Minkowski space of special relativity as two isomorphic interpretations of the quantity of quantum information. Thus a wave function is equivalent to a world line under the condition of wave-particle duality. Both are values of quantum information or "results" of computations of the universe quantum computer.

Intuitively, the two aspects of wave-particle duality should represent correspondingly the two kinds of motion: quantum (discrete) and smooth (continuous). Mathematically, the same is represented by a Fourier-like exchange of the argument of the function: $\frac{1}{t} \leftrightarrow t$, or $E \leftrightarrow t$. The formers are for the quantum representation or interpretation, and the latters are for the smooth ones.

However, one should add that " $\mathrm{t}$ " is for 'time', one rather extraordinary and even unique physical variable: unlike any other, it is featured by its "arrow". This expresses some process of ordering and 
thus it is connected to the concepts of choice and information, and mathematically to the axiom of choice and the well-ordering theorem, which is equivalent to it. Indeed all past is well-ordered by the parameter of time, all future is still unorderable in principle in the present moment, which is between them and in which the ordering is being made by means of choices and thus involving the quantity of information for the choices to be measured as an amount.

Furthermore one can question about that kind of description of reality, which is invariant to the past, future, and present: in other words, that kind of mathematical formalism, which can equate the special variable of time to any other physical quantity, which is not featured by its "arrow". This would require the past, which is always well-ordered, and the future, which is always unorderable in principle, as well as the present, in which the ordering is being made by a relevant series of choices, to be somehow equated to each other. That kind of mathematical formalism is necessary to involve the axiom of choice in a rather extraordinary way: Indeed the present making the ordering requires the axiom of choice in order to be able to convert the unorderable future into the well-ordered past. However the future excludes the axiom of choice, otherwise it would not be "unorderable". Consequently, the searched formalism should possess a property rather contradictory at first glance: invariance to the axiom of choice. It should describe equally well as the well-ordered past utilizing the axiom of choice as the unorderable future excluding it. It seems to anyone that any formalism satisfying that condition should be inconsistent and thus it cannot exist.

However it exists: and even more, it is very well known and even utilized for it is that of quantum mechanics, Hilbert space. One should demonstrate why quantum mechanics is forced to resolve the problem of how to describe uniformly the past and future as well as to involve an invariance to the axiom of choice.

Indeed quantum mechanics is defined as that science, which describes the system of a macroscopic device and a measured quantum entity by the indications of the former. The ratio of their masses or energies can be accepted as converging to infinity. Consequently, the ratio of the periods of the de Broglie waves correspondingly associated to them will converge reciprocally to zero. Thus the present of the quantum entity will includes a big enough part of the future and past of the device. All this should be uniformly reflected in the indications of the device. This implies the necessity for quantum mechanics to describe uniformly the present, future and past as it does absolutely depends on the indications of the apparatus generalizing them in a form of a common theory.

The same can be demonstrated in another way: Quantum mechanics is forced to resolve the problem of how to describe uniformly continuous (smooth) and discrete (quantum) motions. This requires a present moment, in which the continuous motion is, a past moment, which is the beginning of the quantum leap, and a future moment, which is the end of the leap to be described uniformly in general.

One can also demonstrate in a way independent of the above two arguments that the mathematical formalism of quantum mechanics should satisfy the condition for invariance to the axiom of choice: The theorems about the absence of the hidden variables in quantum mechanics (Neumann 1932; Kochen, Specker 1968) exclude any well-ordering in any coherent state of any quantum system before measurement. However the results of any measurement can be always well-ordered in principle by 
means of the parameter of time. This requires the well-ordering theorem, which is equivalent to the axiom of choice. Nevertheless the coherent state before measurement excluding any well-ordering must be equated to the well-ordered set of results after measurement. This implies the invariance at issue.

In turn, the invariance to the axiom of choice implied by the necessity of a uniform description of the present, past and future implies the concept of choice and ordering, on the one hand, and that of information as that quantity expressing the amount of choices or the degree of ordering, on the other hand.

Intuitively, the concept of information is connected with those of 'choice' and 'ordering' so: Information is some relation of different orderings of one and the same entity or eventually the relation between the absolute lack of ordering (as a special kind or benchmark of ordering) and a given ordering. Any ordering is being made by a well-orderable series of choices. Consequently, the choice should be the "atom" or "unit' of ordering and thus that of information. Then the quantity of information should be the quantity of choices necessary to be obtained that ordering at issue or the information interpreted as complexity after Kolmogorov (1968).

Indeed the unit of "classical" information, the binary digit or bit, is an elementary choice between two equiprobable alternatives. Any other finite choice defined as a choice between a finite set of nonequiprobable alternatives can be measured as some real number of bits. Furthermore, any finite series of finite choices can be measured in the same way and represented as the ultimate tape after a Turing machine (Turing 1937) has done its work.

However, that way is irrelevant as to an infinite choice or an infinite series of choices: It does not allow of defining or comparing the quantity of information in infinite sets. Even more, if the concept of infinite choice and corresponding information is introduced, the foundation of set theory will need perfection: The axiom of choice is necessary in order to guarantee always the possibility of choice of an alternative among an infinite set of those. Furthermore, the invariance to the axiom of choice as above is unavoidable for the state before choice, necessary for the concept of information as a relation to it, to make sense. After those conditions have satisfied, the concept of information relevant as to infinite sets can be already introduced and demonstrated that it is isomorphic to that of quantum information in quantum mechanics.

Indeed utilizing the "ball representation" of a qubit, it can be defined as the choice of two points correspondingly the one being among the points of the unit ball, and the other among only the unit sphere (the surface of the unit ball). This means two choices correspondingly among a set and among a true subset of it. Only infinite choice defined as above can be decomposed into two choices, the one of which being among a true subset of the alternatives of the other; and vice versa: if that condition is satisfied, the choice is necessary infinite. And what is more important: the unit of a qubit allows of defining and comparing the quantity of information as to infinite sets. The qubit as the measure of infinite information hardly is unique, but probably it is one of the simplest ones and what is used in nature according to the contemporary corpus of knowledge.

After the concept of quantum information has involved, Hilbert and Minkowski space can be seen also uniformly as two aspects or "hypostases" of quantum information, correspondingly the global and 
local ones. Indeed, on the one hand, any wave function (a point in Hilbert space) can be represented as an infinite series of qubits as follows:

Given any point in (complex) Hilbert space as a vector $\left\{C_{1}, C_{2}, \ldots C_{n}, C_{n+1}, \ldots\right\}$, one can replace any successive couple of its components such as $\left(\left\{C_{1}, C_{2}\right\},\left\{C_{2}, C_{3}\right\}, \ldots\left\{C_{n-1}, C_{n}\right\} \ldots\right)$ with a single corresponding qubit $\left\{Q_{1}, Q_{2}, \ldots, Q_{n}, Q_{n+1}, \ldots\right\}$ such that:

$$
\alpha_{n}=\frac{C_{n}}{(+) \sqrt{\left|C_{n}\right|^{2}+\left|C_{n+1}\right|^{2}}} ; \beta_{n}=\frac{C_{n+1}}{(+) \sqrt{\left|C_{n}\right|^{2}+\left|C_{n+1}\right|^{2}}}
$$

if $C_{n}, C_{n+1}$ are not both 0 . However if both are 0 , one needs to add conventionally the center $\left(\alpha_{n}=0\right.$, $\beta_{n}=0$ ) to conserve the mapping of Hilbert space and an infinite qubit tape to be one-to-one.

On the other hand, any qubit (meaning its "ball representation") is isomorphic to an inertial reference frame after the unit ball with two chosen points from it has interpreted as the space-time ball within the light cone with the position and velocity of an inertial reference frame. The qubit will be one and the same as to all points (the world line) of a reference frame as far as it is inertial. Furthermore, any world line being smooth can be described as an infinite set of its tangents in any point of it. Any tangent of those will represent the world line of an inertial frame and thus a qubit. Consequently, any world line can be mapped one-to-one as a wave function by the mediation of a series of qubits: or in other words, both wave function and corresponding world line can be interpreted as one and the same value of quantum information measured by qubits.

The difference between a world line and its corresponding wave function consists only in the different interpretation of time as this is described above: The world line is being outlined just now, in the present moment distinguishing unambiguously between the well-ordered past and the future unorderable in principle. The present is between them making the choice and therefore transforming the non-orderable future into the well-ordered past. Thus the concept of world line shares only the one interpretation of time. The other interpretation of time generates the concept of wave function describing uniformly the well-ordered past, the ordering present, and the unorderable future by the mediation of the invariance to the axiom of choice. However the concept of world line needs exactly the axiom of choice rather than that invariance to it. Nevertheless the necessity of the axiom of choice is also involved in the invariance to it. The world line describes locally the process of choice while the wave function does the same globally. If one utilizes the representation of the universe as a quantum computer, the distinction between 'world line' and 'wave function' can be seen as that between the current state of a quantum computation and the ultimate result of it.

However if one unifies the concepts of wave function and world line on the base of quantum information, the pathway for quantum mechanics and general relativity to be unified is already outlined clearly in a rather unexpected way: In a sense, they does not need and cannot be unified being one and the same only seen in different aspects: globally and locally.

However, the global aspect should be referred to the smallest while the local aspect to the big and biggest: the macroscopic world and the universe. This contradicts common sense linking the global to the biggest, and the local to the smallest. One way to be reconciled is the conception of the cyclic universe, in which the biggest is returned as the smallest. The discussion till now is consistent to that 
understanding as it is still kinematic for the concept of mass at rest has not yet been introduced. Indeed quantum complementarity is consistent to that "relativity" of the small and the big for any pair of conjugate quantities in quantum mechanics shares it. The mass at rest is what involves certain asymmetry between any two conjugates and therefore between the big and the small.

At last, one can investigate also uniformly the deformations of Minkowski space (as the pseudoRiemannian space of general relativity) and of Hilbert space (as the phenomena of entanglement) being correspondingly the local and global aspect of one and the same generalized deformation.

Indeed, after general relativity has identified any world line in Minkowski space with the corresponding geodesic line in pseudo-Riemannian space, quantum information can identify not only a world line with a wave function, but furthermore any two successive qubits of a world line or a wave function with two parallel qubits of two different world lines or wave functions. Thus entanglement and the gravitational field can be seen uniformly.

If a reference frame in Minkowski space is not inertial, it can be decomposed into two or more inertial reference frames situated successively in time. To be considered the case of two ones is enough for the principle to be visualized. First, these two qubits can be identified as one and the same in a deformed space like pseudo-Riemannian space. Then they can be interpreted as two interacting qubits belonging to the wave functions of two entangled quantum systems. At last, these three cases can be identified. As general relativity identifies inertial mass (the first case) with gravitational mass (the second case) as quantum information identifies the well-ordered time in general relativity (the second case) with the time invariant to ordering in quantum mechanics (the third case). Indeed the inertial mass and the well-ordered time are counterparts by the mediation of energy and quantum duality as well just as the gravitational mass and the time invariant to ordering are the same ones.

By the way, energy (and therefore mass) can be discussed as the "reciprocal space-time" from this viewpoint, which is that of quantum mechanics: Furthermore if special relativity unifies space and time "proportionally", general relativity continues unifying space-time and energy "reciprocally" from this viewpoint originating from quantum mechanics and therefore consistent to it. In other words, the concept of energy (and therefore that of mass) serves to describe the relation between two "kinds" of time: the time-now, which is well-ordered, and the "eternal" time, which is invariant to ordering. The former should be refer to the local aspect and thus, to general relativity and the latter to quantum mechanics and information.

Thus a common viewpoint to the notion of mass at rest in general relativity, quantum mechanics, and the Standard model can be build.

\section{The Standard model in terms of quantum information: mass at rest}

The core of the Standard model is the unity of three symmetries [U(1), SU(2), SU(3)], which "gauge" Hilbert space underlying mathematically quantum mechanics correspondingly as to each one from the following three fundamental interactions: electromagnetic, weak, and strong. Additionally, the three symmetries are independent of each other, or in other words, "orthogonal" to each other so that the Standard model can be represented as the tensor product of them: [U(1)] X [SU(2)] X [SU(3)]. The mass at rest appears by the "Higgs mechanism": $\mathrm{U}(1) \rightarrow[\mathrm{U}(1)] \mathrm{X}$ [SU(2)]. In fact the "Higgs 
mechanism" includes two hypotheses: (1) It explains how the particles of the weak field (Higgs 1964) acquire their mass at rest (2) It shows that all the rest having some nonzero mass at rest should obtain mass by means of the same mechanism.

No one has managed to find that symmetry, which would suitable for gravitational interaction and should complement the Standard model to the cherished "Great unification". The efforts continue. Many scientists suspect that the formulation of the problem is wrong, though. This paper also will try to demonstrate where could be the mistake if the problem of the "Great unification" is formulated as revealing the symmetry relevant to gravity: even more, the mistake of all attempts to be created the theory of quantum gravity.

The objectivity now is to be given a clear and simple interpretation of those symmetries and thus of the corresponding fundamental interactions on the base of wave-particle duality by means of the concepts of quantum information:

The introduction of gauge symmetries addresses the description of the "forces" and "fields" of classical mechanics and physics in a relevant and quantum way. Indeed any classical force forces the motion of any particle undergone to its action restricting all possible positions and states of the particle at issue to a single one. An alternative way for this to be described is the introduction of some suitable "gauge" group relevant to that force so that all possible positions and states of any particle undergone to the action just of this kind of force to be represented as some group and thus as some symmetry unifying all those opportunities in a single mathematical structure. Even more, if the case is quantum mechanics and thus wave-particle duality is granted, the only way to be described mathematically the force is by means of some relevant group and symmetry: Indeed all elements of the group correspond to the wave "half" of duality; any element chosen somehow, for example by measurement, corresponds to the particle "half". However all group and the chosen element are identified by the viewpoint of quantum mechanics just for wave-particle duality. In other words, the laws of quantum mechanics should be invariant to choice.

Classical physics in general and any force in particular are not invariant to choice, though: Any kind of force in classical physics is defined just as that choice, which restricts to the single position and state, which are observed. Emphasizing the differences between the quantum and classical concept of 'force', one can say that the quantum measurement should be a "force" according to the classical understanding. Thus many misinterpretations of quantum mechanics as "subjective" or ostensibly depending on an observer can be clearly elucidated.

Wave-particle duality and the invariance to choice are forced for resolving the main problem of quantum mechanics: how to be uniformly described both the smooth motions of classical physics and new discrete motions in the quantum world commeasurable with the Planck constant. Thus quantum mechanics introduced implicitly a generalization of Einstein's general principle of relativity (e.g. Einstein 1918): All physical laws should be invariant to any motions including the quantum rather than to smooth ones having an exactly determined velocity in any point of the trajectory.

The three gauge symmetries of the Standard model mean the invariance to the choice of an inertial reference frame among the group of the "wave" of the chosen reference frame. Thus all reference frames from the group share a few common parameters: a common space-time, a common position as 
the beginning and a common velocity. All fundamental particles of the Standard model describes the parameters of that chosen inertial reference frame moving smoothly in the dual and thus equivalent terms of a discrete (quantum) motion. Roughly speaking, the fundamental particles are the "coefficients of decomposition" of just that smooth inertial motion in the equivalent representation in terms of quantum motion.

Consequently, there is a privileged subgroup of the group of all inertial reference frames according to the Standard model. The common parameters of their motions are usually masked and designated as the "Bing Bang" put the beginning of the universe. At first glance, this is an obvious and direct contradiction ostensibly both with special and with general relativity elucidating their inconsistency in relation to quantum mechanics mentioned in the beginning: The concept of ether rejected by special relativity is equivalent to some privileged nonempty subset among the set of all inertial reference frame. Though Einstein himself showed (1920) that general relativity unlike special relativity is independent of the concept of ether (i.e. it is consistent both with the concept of ether and its rejection), the privileged reference frames did no return back in physics.

However the Standard model implicitly introduces privileged reference frames even in the fundament of the physical laws by means of the set of particles expressing the parameters of that subgroup of inertial reference frames in quantum language.

The sense of the general "gauge" symmetry underlying the Standard model [U(1)XSU(2)XSU(3)] is the following: It consists of three rotations, which can be considered as independent of each other for the tensor product of them is constituted. These rotations translate the wave-particle duality of quantum mechanics into the language of group symmetry. They mean that the corresponding "wave image" of a chosen reference frame should be accepted as equivalent to each other. The idea of all gauge symmetries is just the theory to be "gauged" to accept the quantum wave as a whole identical to any "point" of it. Furthermore, after the gauge symmetries are "extracted" and determined, all other differences rest on some real differences of the states of one or more quantum systems. Thus after the gauge symmetries are determined, a generalized, "wave-particle" reference frame is chosen so that all differences in quantum states can be defined absolutely to it. This is what is well-known as the "Big Bang". Indeed the concept of the "Big Bang" privileges a reference frame determined by its position $\left(x_{0}, y_{0}, z_{0}\right)$ in the "beginning of time" $\left(t_{0}=0\right)$ as well as its velocity and eventually acceleration: those of the expansion of the universe. However, the Standard model needs a reference frame to be privileged for it to be able to describe the parameters of that reference frame. The "Big Bang" hypothesis is a much stronger interpretation, which is consistent not only to it, but also to a considerable part of the traditional metaphysics involving some Creator, Who has created the universe, etc. A reference frame to be privileged is consistent to general relativity, too.

Now the objectivity is the kind of that reference frame privileged by the Standard model to be restored on the base of the generalized "gauge" symmetry [U(1)XSU(2)XSU(3)]. It consists of three rotations: U(1), SU(2), SU(3). The sense to be rotations has already elucidated above: This involves that invariance necessary for wave-particle duality. The dimensionality of the symmetries is successive beginning from the lowest, which is possible: that of a unit circle, U(1). The next one, SU(2) is that of a unit ball or "3-circle", and the next one, SU(3) is that of a unit "3-ball" or "4-circle", which is 
topologically isomorphic to the space-time of special relativity, i.e. to the so-called imaginary area of Minkowski space. One might suggest that no reason for that kind of circle-symmetries to be restricted to the last known level (i.e. to SU(3) and "strong interaction") and consequently, they might be continued in future in the course of cognition.

In fact, the tensor product of the three symmetries involved by the Standard model to "gauge" the universe quantum-mechanically determines as the privileged reference frame an inertial one consistent to a definite space-time position of the alleged "Big Bang" and a constant velocity of the expansion of the universe. However it cannot determine which exactly should be that inertial reference frame at issue in the Standard model.

There is a fundamental correspondence by means of those three symmetries: U(1) juxtaposes all positions in space-time (which cannot yet be introduced) as equivalent for the light cone is isotropic. Furthermore SU(2) juxtaposes a special position in space-time to weak interaction (the Higgs mechanism) therefore generates the space itself as it needs also some special position in it anywhere be it. At last $\mathrm{SU}(3)$ juxtaposes a special velocity to strong interaction therefore privileging an inertial reference frame, to which any motion can be defined absolutely, and the space-time itself is ultimately constituted as it needs some special (inertial) reference frame in it whichever be it. Consequently spacetime can be considered as a common premise of the Standard model being equivalent to the underlying symmetry: U(1)XSU(2)XSU(3). However, the Standard model also interprets that condition exacting which is the privileged inertial reference frame. The "Big Bang" hypothesis précises further the Standard model attaching that privileged inertial reference frame to the "creation" of the universe, but the Standard models needs exactly this reference frame to be privileged somehow and however. As the Standard model fills the "blank" remained by the underlying symmetry, as the "Big Bang" hypothesis fills an analogical blank about which is the reason an inertial reference frame to be privileged.

No additional symmetries after SU(3) are possible if the contemporary quantum mechanics based on Hilbert space is granted for it is necessary linear and thus excludes from consideration any noninertial reference frame.

In the present context, which can be designated as temporal, the eventual non-linearity of quantum mechanics would mean some kind of interaction between different moments in one and the same time (one and the same well-ordering) or between different "times" (i.e. different well-orderings) in different universes possibly smoothly transiting into each other. Till now, there are no experiments or facts, which might justify that complication and "Occam's razor" removes that possibility of nonlinear quantum mechanics.

The structure of the contemporary fundamental physical knowledge consists of two parts, correspondingly linear and non-linear, which are divided into two disjunctive theories: quantum mechanics and general relativity. The Standard model defines a privileged reference frame, which is consistent to both, but it is necessary inertial and thus only to quantum mechanics. It is hardly amazing for quantum mechanics rather general relativity underlies the Standard model, which in turn is not able to incorporate general relativity in any way.

One can coin the metaphor of a peculiar equation, which should equate quantum mechanics (plus the Standard model) with general relativity just as the linear and non-linear part of an algebraic 
equation therefor implying the possibility of resolving just for some unknown reference frame. The "Big Bang" reference frame satisfies that equation being necessary linear in the singularity of the "beginning of all beginnings" generating the Standard model, and after that transforming smoothly into non-inertial reference frame of the expanding universe according the astronomical observations consistent to general relativity.

However, one can offer a fundamentally different reference frame satisfying the same "equation" therefore explaining otherwise its privileged position: the space-time position of the Earth in the universe. Globally, it is not inertial, but locally it is inertial for the change of its position is too slow in comparison to the measures of human cognition. In other words, the Standard model is valid for about fifty years as the change of the space-time position of the Earth during that period of time is negligibly small.

Nevertheless whether the "Big Bang" or the position of the Earth is what privileges an inertial reference frame, both need the condition of the mass at rest in the Standard model and general relativity to be one and the same or at least commeasurable concepts in order that equation to be able to be constituted. Otherwise one or more additional members of that "equation" should be added or even that "equation" cannot make sense. The unity of the concepts of "mass at rest" is the key.

Though the Standard model involves privileged reference frames, any contradiction to special relativity can be avoided utilizing the above "invariance to choice" "in opposite direction": In the former direction, it means that any group (symmetry, "force") can be replaced equivalently by any element of it. Consequently in the latter direction, it should mean that any element can be equivalently replaced by any group, to which it belongs. In fact those "two directions" have a clear and intuitive sense correspondingly of the direction "from a wave to a particle" and of the direction "from a particle to a wave" in wave-particle duality, which underlies both.

Now the direction "from an element to a group (symmetry, "force")" allows that subgroup of reference frames privileged by the Standard model to be replaced equivalently (i.e. identified) as the entire group of all inertial reference frames therefore restoring special relativity as to the Standard model.

As to general relativity, yet Einstein (1920) himself demonstrated that it is consistent with any privileged reference frames. Consequently, if it is not consistent with the Standard model, the cause cannot be the fact that it privileges some reference frame. However in a sense, the cause could be that the privileged reference frames are inertial. Indeed one can figure that the inertial frames privileged by the Standard model are only a kind of approximation to some non-inertial reference frames, which should be privileged by the future development of the theory. "Dark matter" and "dark energy" can be easily interpreted in thus: the dark matter meaning some positive acceleration of the privileged reference frame would introduce new groups (symmetries) of unitary matrices and thus would enrich the Standard model with new fundamental bosons and fermions, and most of them would have some nonzero mass at rest. Thus that positive acceleration generates the "dark energy" of energy infusion, and the mass at rest of the necessary new particles does the "dark matter". By the way, the dark energy and matter are calculated by means of general relativity therefore demonstrating that 'mass at rest' is not one and the same in both theories (the Standard model and general relativity) as quantities. 
The conceptual analysis shows that the notions of 'mass at rest' are different in both theories rather than some only quantitative difference in their forecasts. In other words, "dark matter" means not only some hidden amount of mass at rest but the quantity of the difference between two ways the concept of 'mass at rest' to be defined in both theories. One can add a third way to be defined for quantum mechanics because it is not exactly the same of the Standard model: Even more, it can serve as a mediator between those two ways for defining 'mass at rest' coining 'entanglement' studied by quantum information.

All three theories define 'mass at rest' as some ratio between two measures of the relation of two inertial frames (or subgroups of such ones having empty intersection).

The Standard model has advantage of giving an absolute definition of 'mass at rest' using two privileged reference frames: that of the "Bing Bang" and that of light. The Higgs mechanism of spontaneous symmetry $\mathrm{U}(1)$ violation $[\mathrm{U}(1) \rightarrow[\mathrm{U}(1)] \mathrm{X}[\mathrm{SU}(2)]$ means a point in all space-time, which has yet been absolutely coherent for U(1), to be chosen, namely that of the "Big Bang". Unlike special relativity, the Standard model has no troubles to attach an inertial reference frame to light for even the usual "non-light" inertial frames in it are defined as relevant subgroups, i.e. invariantly to the rotations of the corresponding unitary matrices. After both special inertial reference frames can be well-defined, they define at once in turn 'mass at rest' and quantities, which can feature its amount: the so-called "God particle", the Higgs boson. Using the same kind of "Bible metaphors", one can say that the "God particle" is absolutely defined by two reference frames: God's reference frame of light and the reference frame of the creation designated by the Creator by the Divine Act of a Point in SpaceTime to Be Chosen as That of The Beginning, the "Big Bang".

By the way, Leon Lederman who wrote (with Dick Teresi) the book "The God Particle: If the Universe Is the Answer, What Is the Question?" (1993) comments the relation between the Standard model and quantum information so:

One of the more intriguing places where quantum spookiness has arisen is in the very creation of the universe. In the earliest phase of creation, the universe was of subatomic dimensions, and quantum physics applied to the entire universe. I may be speaking for the masses of physicists in saying that I'll stick to my accelerator research, but I'm mighty glad someone is still worrying about the conceptual foundations of quantum theory.

For the rest of us, we are heavily armed with Schrödinger; Dirac, and the newer quantum field theory equations. The road to the God Particle — or at least its beginning - is now very clear ( $\mathrm{p}$. 188).

Unlike the Standard model, quantum mechanics cannot initially even define 'mass at rest' at all rather than cannot define mass at rest absolutely. In fact, the concept of 'mass at rest' contradicts the Heisenberg principle of uncertainty if Einstein's $\mathrm{E}=\mathrm{mc}^{2}$ is granted: 'Mass at rest' requires both an exact energy (mass) and an exactly determined moment of time, in which this mass is just "at rest". However the concept of "mass at rest" is consistent with energy conservation, but both contradict that quantum uncertainty. The decision was "Solomonic": The principle of uncertainty was suspended as to energy and time. The so-called "fourth uncertainty" was effectively rejected (Broglie 1990: 273-276) thus 
allowing both energy conservation and mass at rest in quantum mechanics. In fact, 'mass at rest' was implicitly defined in quantum mechanics just by that "Solomonic decision". One can see how:

Quantum mechanics resolves the problem of how the quantum (discrete) and smooth motions to be described uniformly. However this is impossible in any finite mathematical structure: Thus the complex Hilbert space is involved. It is not only infinitely dimensional explicitly, but also any finitely dimensional subspace of it uses implicitly infinity for its basis is complex. Its units can be equivalently represented as qubits. Therefore quantum mechanics is always linear, and the underlying mathematical formalism of the infinitely dimensional complex Hilbert space is correspondingly always "flat" in a sense. However, only a single and standalone quantum system can be described in thus in general. In particular, an arbitrary number of subsystems can be done in the same way as far as they are associated with subspaces, including infinitely dimensional, of the Hilbert space of the system. Mass at rest serves for this to be always possible.

If the compound system consists of two entangled subsystems, this implies some nonzero mass at rest of the system conserving the general principle of orthogonality: a joint space for the system and its subspaces for the "particles". Indeed then mass at rest is introduced relatively as the ratio of two measures of the orthogonal subgroups having an empty intersection as being orthogonal to each other. In fact, all entanglement is represented globally as a correctional coefficient of mass at rest. After it has introduced, the corresponding subspaces can be disentangled. This is the way for the "particle" ideology to be established in quantum mechanics and developed further in the Standard model, which defines the mass at rest already absolutely, as above. Consequently, the concept of mass at rest and that

of entanglement are complement in a sense. Entanglement cannot be consistently introduced in the Standard model directly just as it is always introduced implicitly by means of "mass at rest" representing entanglement integrally and globally.

One can see a little below that general relativity introduces mass at rest in a third way, which is both local and relative. General relativity cannot be incorporated in the Standard model explicitly (the cherished theory of quantum gravity or the "Great unification") just because it is already incorporated implicitly by the concept of mass at rest and by the "particle" ideology. In other words, the Standard model is as "complement" to general relativity as it is "complement" to quantum information above. If the Standard model defines mass at rest absolutely and globally, general relativity does the same relatively and locally. The "bridge" between them is the theory of entanglement, i.e. quantum information, by defining mass at rest relatively as general relativity, but globally as the Standard model. Furthermore it constructs a relevant isomorphism between the global and local representation. So, one can return more backward in history: from the Standard model through quantum mechanics back to general relativity, the teenage years of the $20^{\text {th }}$ century.

\section{General relativity in terms of quantum information: mass at rest}

General relativity defines mass at rest as the ratio of the measure two subgroups of inertial frames: that of space-time in the point, in which the particle with nonzero mass at rest is, and that of this particle. The space of general relativity is smooth almost everywhere and thus it is locally "flat" there: that is the subgroup of inertial frames in the point can be replaced by the corresponding subgroup in the 
"flat" Minkowski space. In other words, the geodesic line can be replaced equivalently by its tangent in a neighborhood of the point. The subgroup of inertial frames "of light" both in pseudo-Riemanian and Minkowski space can be defined just in the same way as quantum mechanics does it.

Nevertheless, general relativity is not able to define 'mass at rest' absolutely, as the Standard model manages to do this globally, even locally though it is both consistent with privileged reference frames as Einstein showed and having the one subgroup of the privileged reference frames: that of light in vacuum. The cause is that it does not privilege any other subgroup and thus is not able to determine any privileged relation and ratio. However it being consistent with privileged reference frames can be complement with a such one if one manages to define it relevantly at least as much as the Standard model does this.

\section{Mass at rest in the Standard model as an interpretation both in quantum information and general relativity}

The following is necessary to be demonstrated:

Quantum information and general relativity define mass at rest equivalently but from different viewpoints: correspondingly globally and locally in relation to time.

Both are consistent not only to each other but also to the Standard model, which complements them with a privileged relation and ratio by means of correspondingly a privileged subgroup of inertial frames attachable to the "Big Bang" or to the Earth and a privileged wave function attachable to the universe. However both privileged viewpoints should be equivalent according to the former statement.

In other words: The local viewpoint in relation to time generates the concept of a privileged reference frame, which can be approximately or exactly inertial as the Standard model states in addition, or non-inertial as the abundance of "dark matter" or "dark energy" hints. The global viewpoint in relation to time generates the concept of a wave function. Both viewpoints are equivalent to each other. However both add a fundamental random choice determining physical reality by means of some fundamental constants.

The nature of that fundamental choice needs some philosophical interpretation, which turns out to be different in each of both cases somehow "by itself":

The metaphor of the "Big Bang" suggests the choice to be external to the universe and thus all series of "Bible metaphors" takes place.

The concept of the privileged wave function of the universe suggests that the choice is made in the present moment of time by some real observer such as mankind on the Earth. It implies some changes in the fundamental constants and even in the form of the physical laws not only in the course of time but even from an observer (i.e. reference frame) to another in the universe. Being too shocking, this conclusion should be purposely emphasized: physical reality can be different in relation to different observers in the universe. In particular, the Standard model, even only approximately, is valid in a limited neighborhood of the earth though too vast in order to be commeasurable with the dimensions of the universe. Another position can privilege another wave function and thus another reference frame, another "Big Bang" and other laws of the physics. Though the universe is one single, it can seem 
radically different to remote enough observers. Furthermore, the laws of physics can change in the course of time even on the Earth.

Consequently, the "Big Bang" interpretation implies some common metaphysics shared by all observers in the universe: It includes a common beginning of the universe, namely the "Big Bang", as well as a common corpus of physical laws, namely the "Standard model" and any future perfections of it. It is a last form of geocentrism naively suggesting that the physical laws valid on the Earth are necessary valid anywhere in the universe as if the Earth should be the center of the universe by itself.

The concept of the "here-and-now" choice bids farewell to "God" and thus abandons any universal metaphysics in the universe in the following sense: The choice depends on the space-time position, in which it is made. It will define different referent frames and different wave functions as privileged according to the corresponding space-time position. This will imply different "Standard models" in remote enough points of the universe. The transition between two "Standard models" would be smooth. Two "Standard models" can be considered as two "universes" or "worlds" in the many-world interpretation of quantum mechanics. However the main problem of quantum mechanics is how any smooth transition and discrete leap between two states (e.g. two space-time positions) to be described mathematically uniformly. After it has resolved that problem, the solving implies that two "parallel" universes can be also seen as two remote enough observers within a single universe like ours. Even more, their different corpuses of fundamental physical laws are unambiguously determined by their corresponding space-time positions (reference frames) to the universe. Looking at the universe, the observers in each of them will see quite different phenomena for the fundamental physical laws will be quite different in each reference frame. The unification of special and general relativity and quantum mechanics is what implies that generalization of the dependence on the observer in the former two theories. The concept of observer or observation in the latter theory is replaced by that of measurement. Any quantum measurement orders the universe differently, and the many-world interpretation of quantum mechanics situates any different ordering as a different "world" or as a "parallel universe". The unification with relativity, which requires smooth transitions almost everywhere in the universe, is what implies that the space-time position determines the laws (the ordering) in the "world" (the "parallel universe") of any remote enough observer.

What is necessary to be demonstrated at last is how quantum information and general relativity define mass at rest equivalently. Quantum information can define mass at rest integrally, to all time, while general relativity does the same differentially, to any given moment therefore requiring for the change in time to be smooth.

\section{Conclusions about the mathematical fundament of the physical world}

One can offer the following thought experiment: A spaceship starts from the Earth to some star in a nebula, which is remote enough. It would be better the acceleration of the spaceship to exceed that of the expansion of the universe. It will continue to seem as a spaceship to any observer on Earth according to the physical laws valid on it. However an observer in the spaceship will find that the fundamental physical laws or constants are changing gradually in its course. 
The unification of quantum mechanics and general relativity, which implies that mass at rest is one and the same in both cases, implies also that dependence of the physical laws of the space-time position of the observer. The universe will be as that elephant from the parable, which is described by blinds quite differently according to which part of the elephant they touch. Their description is absolutely dependent on their positions to the elephant.

This implies a rather extraordinary relation between the set of different physical realities according to the different observers, on the one hand, and a common or at least a more constant mathematical form underlying them, on the other hand. Those realities depending on the observers are only "outward appearances", the mathematical form of the underlying laws is universal. However the transition between the mathematical form and its different phenomena is gradual. The concept of quantum information serves to describe the link of the mathematical and physical as well as that between general relativity and quantum mechanics.

\section{References:}

De Broglie, L. (1990) Heisenberg's Uncertainties and the Probabilistic Interpretation of Wave Mechanics with Critical Notes of the Author, Dordrecht/ Boston/ London, Kluwer Academic Publishers, (Chapter 16).

Einstein, A. (1918) "Prinziplelles zur allgemeinen Relativitätstheorie," Annnalen der Physik 55 (4): 241-244.

Einstein, A. (1920) Äther und Relativitätstheorie. Berlin: Springer.

Einstein, A., Podolsky, B., \& Rosen, N. (1935) "Can Quantum-Mechanical Description of Physical Reality Be Considered Complete?” Physical Review 47 (10): 777-780.

Higgs, P. (1964) "Broken symmetries and the masses of gauge bosons," Physical Review Letters 13 (16): 508-509.

Kochen, S. \& Specker, E. (1968). "The problem of hidden variables in quantum mechanics," Journal of Mathematics and Mechanics 17 (1): 59-87.

Kolmogorov, A. (1968) Logical basis for information theory and probability theory. IEEE Transactions on Information Theory 14: 662-664.

Lederman, L. \& Teresi, D. (1993) The God particle: If the Universe Is the Answer, What Is the Question? London: Bantam, (Chapter 5).

Neumann, J. von (1932) Mathematische Grundlagen der Quantenmechanik, Berlin, Verlag von Julius Springer, (Chapter IV.2), pp. 167-173.

Turing, A. (1937) "On computable numbers, with an application to the Entscheidungsproblem," Proceedings of London Mathematical Society, series 242 (1): 230-265. 